\title{
Effects of Split Style Olympic Weightlifting Training on Leg Stiffness Vertical Jump Change of Direction and Sprint in Collegiate Volleyball Players
}

\author{
İzzet İnce \\ Department of Sport Sciences, Faculty of Health Sciences, University of Ankara Y1ldırım Beyazit, Turkey
}

Copyright $\bigcirc 2019$ by authors, all rights reserved. Authors agree that this article remains permanently open access under the terms of the Creative Commons Attribution License 4.0 International License

\begin{abstract}
One of the most widely used Olympic weightlifting derivatives, hang snatch and clean, is believed to be one of the most effective ways to improve the performance of athletes in sports that require power, strength, and speed. This study was conducted to investigate the effects of split-style hang snatch, and clean and jerk training (twice a week for $6 \mathrm{wk}$ ) on leg stiffness, vertical jump, agility, and sprint performance in volleyball players. The participants $(n=34$ collegiate female volleyball players) were randomized into training $(n=17$, age $=15.63 \pm 1.3$ years, height $=166.05 \pm 5.7 \mathrm{~cm}$, body weight $=63.45 \pm 2.97 \mathrm{~kg})$ and control groups $(\mathrm{n}=17$, age $=15.23 \pm 1.83$ years, height $=167.46 \pm 5.69 \mathrm{~cm}$, body weight $=60.46 \pm 4.14 \mathrm{~kg}$ ). The variables included spike jump, counter movement jump, time to $5-\mathrm{m}$ sprint and $20-\mathrm{m}$ sprint, change of direction, and leg stiffness. The effects of split-style hang snatch and clean and jerk training on the measured variables were assessed by magnitude-based inferences. Generally, the increases in the measured variables were higher in the training group. The probability of an increase in leg stiffness was very likely (precision, $98.9 \%, \mathrm{~d}=0.722)$; 5-m sprint increase very likely $(99.7 \%$, $\mathrm{d}=-1.544) ; 20-\mathrm{m}$ sprint increase very likely $(99.9 \%$, $\mathrm{d}=-0.774)$; and the probability of increase in change of direction was found to be likely, $(85.4 \%, \mathrm{~d}=-0.385)$. The increases in countermovement jump were insignificant. We conclude that split hang snatch and clean and jerk exercises improve leg stiffness, spike jump, time to 5-m and $20-\mathrm{m}$ sprint, and change of direction in female volleyball players.
\end{abstract}

Keywords Olympic Weightlifting, Weightlifting, Snatch and Clean, Stiffness, Vertical Jump

\section{Introduction}

Volleyball is commonly defined as a sport that requires high speed and explosive activities [1]. Frequent shifts, sprinting, dives, dunks, or block repetitive maximal or close-to-maximum jumps are frequent movements during a volleyball match $[2,3]$. Therefore, effectively developing maximum strength and power is necessary in volleyball. To optimize the competition performance of athletes, it is important to develop maximum strength in the early stages of long-term training and to transfer the maximum strength to power effectively as the competition approaches. Therefore, volleyball players routinely perform strength training to improve performance-based neuromuscular abilities, such as power and strength. However, there is only one expert opinion on the effectiveness of Olympic weightlifting exercises in volleyball strength training [3], and very few studies [4] evaluate the effectiveness of training. For this reason, more research is needed to understand the effect of Olympic weightlifting and derivatives on volleyball performance.

The ability to develop high-level muscle strength is considered to be an essential component of success in many sports activities. Olympic weightlifting exercises are included in the strength and fitness programs of most amateur and professional athletes and are generally considered as a superior strength training method for muscle strength development and athletic performance [5, 6]. This may be because of the biomechanical similarities of Olympic lifts to many sports movements and their effects on larger strength and power characteristics compared with other exercises [7, 8]. Although some disagreements exist between exercise professionals in relation to their transferability to sports performance, because of the complex nature of Olympic weightlifting movements, [9] evidence [4, 8] indicate that Olympic lifts are effective strength training method to increase athletic performance. Compared with many variations of Olympic weightlifting, the hang positions of the snatch and clean are considered as "power positions". The highest peak power output and ground reaction forces have been known to 
occur during the explosive second-pull phase (triple extension from the middle thigh, also called the hang position) [10-12]. To the best of our knowledge, the performance results of split-style hang power snatch and clean and jerk (SW) exercises have not been investigated in any study, although there is widespread belief in the benefits of Olympic weightlifting exercises and variations. Therefore, the aim of the study was to address this gap in literature on SW exercises. Even if the classical-style hang snatch and clean $(\mathrm{CW})$ provides a mechanical advantage for Olympic weightlifting performance compared with SW, the mechanical lifting disadvantage of SWs may require a higher power output. In addition, the stepping in the transition from the split to splash is similar to the split motion. Hence, split style Olympic lifts can be effective on the spike jump (SJ) performance, which is very important for volleyball performance. Thus, this study also evaluated the effects of SW exercises on specific volleyball performance.

\section{Materials and Methods}

\subsection{Study Design}

Pre- and post-tests were performed in a rested state (no training $>48 \mathrm{~h}$ before the tests) to evaluate the effects of SW exercises on six performance variables, SJ, counter movement jump (CMJ), time to $5-\mathrm{m}(\mathrm{S} 5 \mathrm{~m})$ and $20-\mathrm{m}$ sprint (S20m), change of direction (COD), and leg stiffness (LS) of female collegiate volleyball players. The participants were randomly divided into two groups: the training (in addition to normal volleyball training, 2 days a week, the SW exercises) and control groups (normal volleyball training only). The training program lasted 6 weeks, and all participants received the same test protocol before and after the training. All measurements were taken in the same environment. Before the tests, subjects warmed up by performing a standard warm-up protocol, including stretching exercises, jogging, and free jumps. After the warm-up, the participants were given a number of attempts to get the tests to be familiarized. Participants were motivated and encouraged to perform well during all tests. All measurements were taken by the same investigator.

\subsection{Participants}

The participants composed of 34 female college volleyball players, who were physically active with similar demographics and activity backgrounds. The descriptive statistics of the participants are presented in Table 1. All participants were training at Ankara Yıldırım Beyazit University Sports Club and had $3.2 \pm 0.37$ years of volleyball experience. Nutritional intake was not controlled; but subjects were asked to maintain their normal diet during the study. By the end of the 6-week training period, two subjects in the control group abandoned the program because of personal reasons. The families of all participants were informed regarding the possible risks and disturbances related to the experimental procedures, and their consent was obtained. The study protocol was approved by Ankara Yıldırım Beyazit University Ethics Committee.

\subsection{Anthropometric Measurements and Body Composition}

The height of each athlete was measured with a stadiometer with $0.01-\mathrm{cm}$ accuracy using standard procedures (Holtain Ltd., Crymych, Dyfed, UK). The body composition was analyzed using a Bioelectrical Impedance Analyzer (BC-310, Tanita Corp., Tokyo, Japan).

Table 1. Descriptive Statistics

\begin{tabular}{ccccc}
\hline & Group & N & Mean & Ss \\
\hline \multirow{2}{*}{ Age (year) } & Training & 17 & 15,63 & 1,30 \\
\cline { 2 - 5 } & Control & 17 & 15,23 & 1,83 \\
\hline \multirow{2}{*}{ Height (cm) } & Training & 17 & 166,05 & 5,71 \\
\cline { 2 - 5 } & Control & 17 & 167,46 & 5,69 \\
\hline \multirow{2}{*}{ Body Weight (kg) } & Training & 17 & 63,45 & 2,97 \\
\cline { 2 - 5 } & Control & 17 & 60,46 & 4,14 \\
\hline \multirow{2}{*}{ BMI (cm $)$} & Training & 17 & 22,97 & 2,90 \\
\cline { 2 - 5 } & Control & 17 & 19,16 & 1,68 \\
\hline \multirow{2}{*}{$\begin{array}{c}\text { Fat Percentage } \\
(\%)\end{array}$} & Training & 17 & 26,83 & 4,41 \\
\cline { 2 - 5 } & Control & 17 & 24,67 & 3,20 \\
\hline
\end{tabular}

\subsection{Leg Stiffness}

Leg stiffness tests were performed based on the protocol applied in a validity and reliability study [13]. Optojump Next ${ }^{\mathbb{B}}$ (Microgate, Bolzano, Italy) Stiffness protocol was applied. Two trials with 2-min rest was applied. The mean contact and flight times from all jumps and participants' body mass, obtained from the resulting vertical force-time trace, were used to calculate leg stiffness. Leg stiffness was calculated using followed "Eq. 1 " by Dalleau et al. [14].

$$
\text { Leg stiffness }=\frac{\text { Mass } \times \pi(\text { flight time }+ \text { contact time })}{\text { contact time }{ }^{2} \times\left(\left(\frac{\text { flight time }+ \text { contact time }}{\pi}\right)-\left(\frac{\text { contact time }}{4}\right)\right)}
$$




\subsection{Spike Jump and Counter Movement Jump}

Subjects were tested for SJ previously established methods by Sattler at al., 2012 [15]. In the SJ test, the subject used an individualized 2- to 3-step approach and performed splashing with arm rotation. This movement followed a vertical upward jump as fast as possible with a strong backward arm rotation. The subjects were asked to perform the jump procedure in a volleyball game or practice, similar to their personal techniques, as they found the most appropriate. The specific procedures were relatively non-standard, as we wanted subjects to use their individual procedures to perform the SJ test. For the CMJ test, the participants were requested to squat and jump vertically as quickly as possible with their hands on their waists, knees at full extension, and bodies upright. Pulling off the knees in the flight phase, pausing during movements, staying out of the Optojump Next ${ }^{\mathbb{R}}$ and the parallel bar range and stepping on the parallel bars were considered failures, and the test was repeated. Two trials were performed for $\mathrm{CMJ}$ and $\mathrm{SJ}$ tests with a 2-min rest in between, and the best result was used in the analysis. CMJ and SJ tests were obtained with Optojump Next ${ }^{\mathbb{B}}$ (Microgate, Bolzano, Italy).

\subsection{Five- and 20-m Sprint Tests}

The participants started the test from the starting line, $1 \mathrm{~m}$ behind the starting photocell, when they felt ready. The measurements were obtained with photocell doors (Microgate, Bolzano, Italy) placed at the beginning and end of the $5 \mathrm{~m}$ and $20 \mathrm{~m}$ run distance. Two-minute measurements were taken at 3-m rest intervals.

\subsection{Change of Direction}

The Standard t-test was used to determine COD ability. The four cones were arranged in a $\mathrm{T}$ shape. To the first funnel starting line, the second cone was placed at $9.14 \mathrm{~m}$ forward, and two cones were placed on the right and left sides of this cone at a distance of $4.57 \mathrm{~m}$. The subjects should sprinted forward for $9.14 \mathrm{~m}$ from the starting line to the first cone and touching it with the tip of their right hand, run a side step with the left hand, move to the second cone that is $4.57 \mathrm{~m}$ to the left, then touch the right cone at a distance of $9.14 \mathrm{~m}$, and touch the middle cone at a distance of $4.57 \mathrm{~m}$. The test was completed with the arrival to the starting line. The timing was determined using a photocell placed on the starting line. Each participant performed two trials to make for reliability purposes. These trials were considered unsuccessful when participants did not contact a designated cone and run smoothly sideways and backward. The test was repeated twice, and the better test time was evaluated.

\subsection{Training Protocols}

During the study, two groups (training and control) participated in standard volleyball training for $6 \mathrm{~h}$ per week ( 3 sessions per week; 120 min per session) for 6 weeks. Standard volleyball training includes technical and tactical volleyball training. Typical volleyball sessions were divided into warm-up, primary, and recovery periods. The warm-up took 20 min and included increased jogging, maximal six upper body exercises (push-ups, etc.), and both upper and lower-body stretching exercises. In the main part of a training session, on-site skills training (attack and defense basics, technical and tactical training, special cases) and real play took place. The work/rest ratio was close to 1:1.

A two-week adaptation program was organized to teach lifting techniques to the weightlifting group. A lightweight training bar made of wood was used in the adaptation program. After the adaptation program, the 1RM (maximum weight that an individual can lift once) was calculated separately using the Brzycki formula: $1 \mathrm{RM}$ $=$ the amount of weight/ $(1.0278-(0.0278 \times$ number of repetitions)) related to each movement [16].

A standard warming protocol was established. The warm-up took $20 \mathrm{~min}$, and included running, stretching exercises, and weightlifting with a weightless and very light training bar. The participants performed a progressive training protocol (2 days/week) during 6 weeks with an increasing intensity rate of $5 \%$ of $1 \mathrm{RM}$ per week from $70 \%$ to $90 \%$. The training program was performed over 3 sets of 5 repetitions in the first week and increased by 1 set during 5 weeks. In the sixth week, the number of sets was reduced to 4 sets of 5 repetitions. (Table 2) There was a 2-minute rest between sets. Each training session contained three movements of Olympic split lifts, hang split snatch and clean and jerk. There were 10-minute resting intervals between three movements, respectively. All athletes were supervised by the same coach and the athletes were encouraged to perform all their movements as fast and explosively as possible.

Table 2. Volume (Set / Repeat) and Intensity Rates of Training Programs

\begin{tabular}{cccccccccc}
\hline & \multicolumn{3}{c}{ Week 1 } & \multicolumn{3}{c}{ Week 2 } & \multicolumn{3}{c}{ Week 3 } \\
\hline & $\mathrm{S}$ & $\mathrm{R}$ & $\%$ & $\mathrm{~S}$ & $\mathrm{R}$ & $\%$ & $\mathrm{~S}$ & $\mathrm{R}$ & $\%$ \\
\hline Hang Split Snatch & 3 & 5 & 70 & 4 & 5 & 75 & 5 & 5 & 80 \\
\hline Hang Split Clean & 3 & 5 & 70 & 4 & 5 & 75 & 5 & 5 & 80 \\
\hline Split Jerk & 3 & 5 & 70 & 4 & 5 & 75 & 5 & 5 & 80 \\
\hline & \multicolumn{1}{c}{ Week } & 4 & & Week & 5 & & Week 6 \\
\hline Hang Split Snatch & 2 & 5 & 85 & 2 & 5 & 85 & 2 & 5 & 80 \\
\hline Hang Split Clean & 3 & 5 & 85 & 3 & 5 & 85 & 3 & 5 & 80 \\
\hline Split Jerk & 3 & 5 & 85 & 3 & 5 & 85 & 3 & 5 & 80 \\
\hline
\end{tabular}

Abbreviations: S: Number of sets, R: Number of repetitions 


\section{Statistical Analysis}

The magnitude-based inference (MBI) method was used for statistical analysis [17]. The probability of a standardized magnitude $(0.35)$ effect on the variables in the pre- and post-test was calculated by Cohen's $d$, and the effect size classification of Rhea [18] for strength training was used. Based on this classification, $<0.35$ points indicate a trivial effect; $0.35-0.80$, small effect; $0.80-1.50$, medium effect; and $>1.50$ was evaluated as the major effect. The differences in the variables were characterized by probabilistic terms, and the following scale was used: $25 \%-75 \%$, possibly; $75 \%-95 \%$, likely; $95 \%-99.5 \%$, very likely; and $>99.5 \%$ most likely. The inference was categorized as uncertain that the $95 \%$ confidence limits (CL) overlapped with the threshold values for the smallest worthwhile positive and negative effects [17].

\section{Results}

The training group performance on SJ (effect size: $d=0.35,95 \%$ CI $=0.039 / 0.678$, mean difference: $1.88 \mathrm{~cm}$;
MBI: possibly positive), CMJ (effect size: $d=0.26,95 \%$ $\mathrm{CI}=-0.16 / 0.61$, mean difference: $0.854 \mathrm{~cm}$, MBI: trivial), LS (effect size: $0.90, \quad 95 \% \quad \mathrm{CI}=0.16 / 1.6$, mean difference $=9.04 \mathrm{kNm}^{-1}$, MBI: most likely positive), COD $(d=-0.385,95 \%$ CI, $0.024 / 0.75$, mean difference: $0.30 \mathrm{~s}$; MBI: likely positive), S20m (effect size: $d=0.814,95 \%$ $\mathrm{CI}=0.48 / 1.2$, mean difference: $0.16 \mathrm{~s}$; $\mathrm{MBI}$ : possibly positive), S5m sprint (effect size: $d=1.544,95 \%$ $\mathrm{CI}=0.92 / 2.2$, mean difference: $0.14 \mathrm{~s}$; MBI: most likely positive) tests improved. In general, the changes in the variables for the control groups were unclear or trivial. No significant changes in SJ (effect size: $d=-0.17,95 \%$ $\mathrm{CI}=-0.421 / 0.760$, mean difference: $1.1 \mathrm{~cm}$; MBI: unclear), CMJ (effect size: $d=0.025,95 \% \mathrm{CI}=-0.414 / 0.463$, mean difference: $0.07 \mathrm{~cm}$, MBI: unclear), LS (effect size: $d=-0.017,95 \% \mathrm{CI}=-0.64 / 0.4$, mean difference $=-0.34$ $\mathrm{kNm}^{-1}$, MBI: trivial), COD (effect size: $d=-0.007,95 \%$ $\mathrm{CI}=-0.36 / 0.37$, mean difference: $-0.07 \mathrm{~s}$; MBI: likely trivial), S20m (effect size: $d=0.045,95 \% \mathrm{CI}=-0.51 / 0.6$, mean difference: $0.15 \mathrm{~s}$; MBI: unclear), S5m (effect size: $d=0.2795 \% \mathrm{CI}=-0.99 / 0.045$, mean difference: $-0.02 \mathrm{~s}$; MBI: trivial) were observed (Table 3) (Figure 1).

Table 3. Pretesting and Post-testing Results [Presented as Means (SDs)], Differences between Measurements, and Magnitude-Based Inference Chances for Positive/Negative/Unclear/Trivial Training Effects

\begin{tabular}{|c|c|c|c|c|}
\hline \multicolumn{5}{|c|}{ Training Group $(\mathrm{n}=17)$} \\
\hline & Pre & Post & Diff & MBI \\
\hline $\mathrm{SJ}(\mathrm{cm})$ & $36.26 \pm 5.44$ & $38.14 \pm 4.88$ & 1,88 & $\begin{array}{c}53.7 / 46.3 / 00 \\
\text { possibly positive }\end{array}$ \\
\hline $\mathrm{CMJ}(\mathrm{cm})$ & $23.83 \pm 3.03$ & $24.69 \pm 3.06$ & 0.85 & $\begin{array}{c}34.9 / 64.5 / 0.6 \\
\text { trivial }\end{array}$ \\
\hline $\mathrm{LS}\left(\mathrm{kNm}^{1}\right)$ & $35.40 \pm 9.50$ & $44,44 \pm 10,5$ & 9.04 & $\begin{array}{c}94.3 / 5.5 / 0.1 \\
\text { likely positive }\end{array}$ \\
\hline COD (s) & $11,92 \pm 0,87$ & $11,62 \pm 0.61$ & $-0,30$ & $\begin{array}{c}58.1 / 41.9 / 10.0 \\
\text { possibly positive }\end{array}$ \\
\hline $\mathrm{S} 20 \mathrm{~m}(\mathrm{~s})$ & $3,75 \pm 0,19$ & $3,59 \pm 0,17$ & $-0,16$ & $\begin{array}{c}\text { 99.5/0.5/0,0 } \\
\text { most likely positive }\end{array}$ \\
\hline $\mathrm{S} 5 \mathrm{~m}(\mathrm{~s})$ & $1,27 \pm 0,10$ & $1,13 \pm 0,07$ & $-0,14$ & $\begin{array}{c}94,3 / 5.5 / 0,1 \\
\text { most likely positive }\end{array}$ \\
\hline \multicolumn{5}{|c|}{ Control Group (n=17) } \\
\hline & Pre & Post & Diff & MBI \\
\hline $\mathrm{SJ}(\mathrm{cm})$ & $36.56 \pm 2.85$ & $37.66 \pm 4.25$ & 1.1 & $\begin{array}{c}35.1 / 51.9 / 13.9 \\
\text { unclear }\end{array}$ \\
\hline $\mathrm{CMJ}(\mathrm{cm})$ & $25.48 \pm 3.13$ & $25.55 \pm 2.33$ & 0.07 & $\begin{array}{c}8.7 / 85.2 / 6.1 \\
\text { unclear }\end{array}$ \\
\hline $\mathrm{LS}\left(\mathrm{kNm}^{1}\right)$ & $35,43 \pm 4,72$ & $35,09 \pm 6,64$ & -0.34 & $\begin{array}{c}3.7 / 79 / 17.4 \\
\text { trivial }\end{array}$ \\
\hline $\mathrm{COD}(\mathrm{s})$ & $12,70 \pm 1,04$ & $12,71 \pm 0,92$ & $-0,07$ & $\begin{array}{c}0.8 / 98.5 / 0.7 \\
\text { trivial }\end{array}$ \\
\hline $\mathrm{S} 20 \mathrm{~m}(\mathrm{~s})$ & $3,69 \pm 0.16$ & $3,70 \pm 0,15$ & 0.15 & $\begin{array}{c}10.4 / 84.1 / 5.5 \\
\text { unclear }\end{array}$ \\
\hline S5m (s) & $1.15 \pm 0.07$ & $1.13 \pm 0,09$ & $-0,02$ & $\begin{array}{c}3.7 / 79 / 17.4 \\
\text { trivial }\end{array}$ \\
\hline
\end{tabular}

Abbreviations: SJ: Spike Jump, CMJ: Countermovement Jump, LS: Leg Stiffness, COD: Change of Direction, S20m: Time to 20m Sprint: S5m: Time to $5 \mathrm{~m}$ Sprint, MBI: Magnitude Based Inferences. 


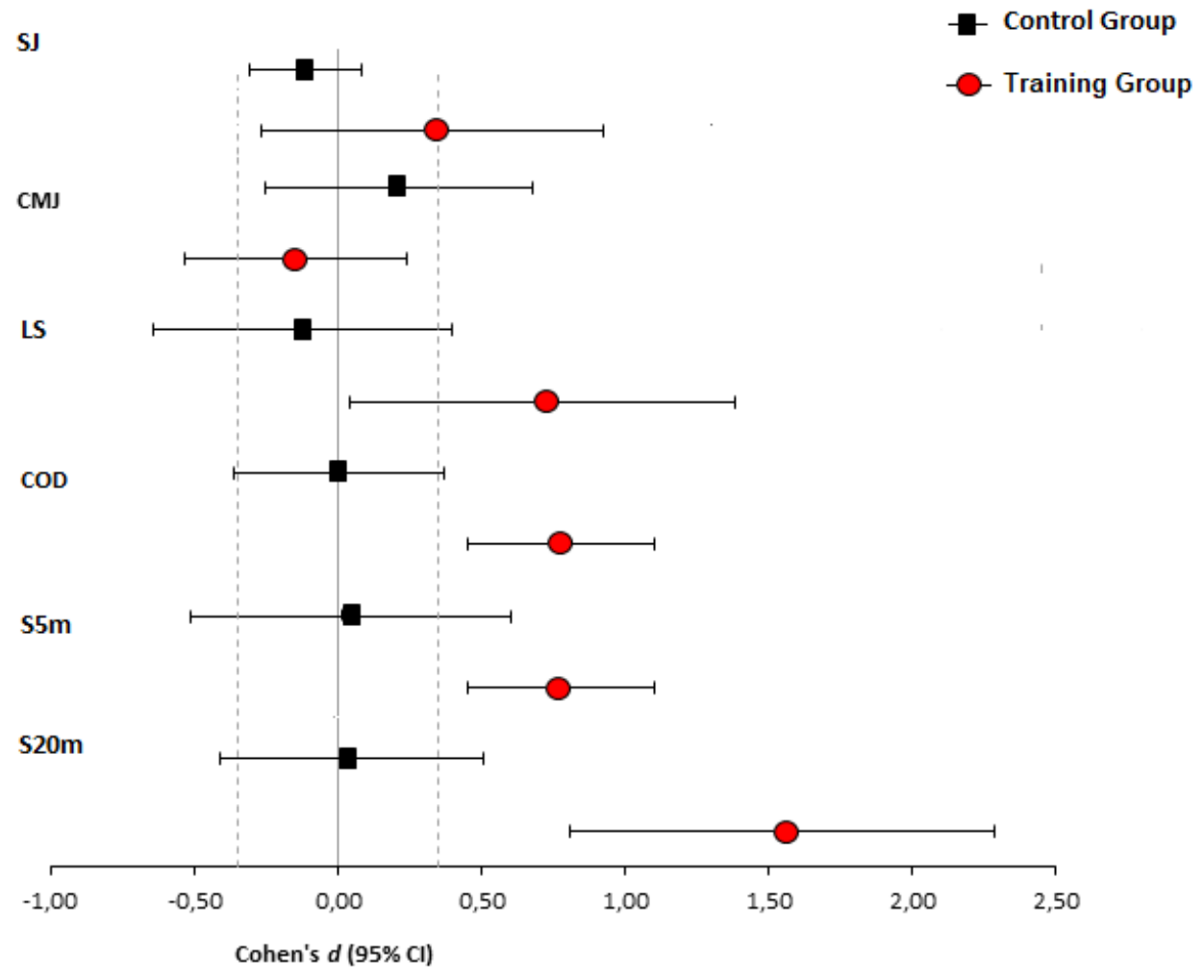

Abbreviations: SJ: Spike Jump, CMJ: Counter Movement Jump, COD: Change of direction, S5m: Time to 5m Sprint, S20m: Time to 20m Sprint.

Figure 1. Magnitude-based inferences for improvement (positive), reduction (negative), or inconclusive (trivial) in all experimental measures. Data are reported as (mean [post-pre] $\pm 95 \%$ confidence limits).

\section{Discussion}

The aim of this study was to investigate the effects of SW exercises on leg stiffness, vertical jump, agility, and sprint performance in female volleyball players. No previous study has investigated the effect of split-style Olympic lift exercises on training performance. Therefore, the results of this study were discussed on the basis of those of previous studies that examined $\mathrm{CW}$ exercises and the effects of other Olympic lifting derivatives. In our study, significant increases were observed in all variables, except for CMJ. Thus, SW exercises do not have a significant effect on CMJ. However, in previous studies, a strong positive relationship was shown between Olympic weightlifting and its derivatives and sports movements, particularly for vertical jump [8, 19, 20]. In previous studies examining the training effect of $\mathrm{CW}$ exercises [21, 22], some important changes have been identified on CMJ. According to Arabatzi et al. [23] Olympic weightlifting exercises seem to improve vertical jump height via changes in power and technique. Ayers et al. [4] observed significant changes in CMJ performance in their study, which compared the training effects of $\mathrm{CW}$ exercises on some variables. A few studies have also shown significant improvements in vertical jump performance on the training effect of some Olympic lift derivatives. [24-27]. In contrast to the mentioned studies Helland et al. [28] reported that OWL training resulted in smaller improvements in CMJ performances. However, although we could not find a study similar to our study, expert opinions [8, 29-33] and biomechanical evaluations [21, 22] argue that there is an important potential for Olympic weightlifting exercises on vertical jump performance. Thus, the reason why SW exercises do not have a significant effect on $\mathrm{CMJ}$ performance can be that $\mathrm{CW}$ exercises have a more similar pattern of motion with the vertical jump. However, owing to the inability to provide adequate adaptation to a 6-week training period on CMJ performance or loading parameters applied in our study, an inadequate adaptation may have occurred. Although no significant improvement was found in CMJ performance in our study, the increase in SJ performance was significant $(d=0.35)$. The last split step before the SJ of SW exercises may improve the power output. In future studies, more explicit relationships may be detected via kinetic and kinematic analyses comparing $\mathrm{SW}$ and $\mathrm{CW}$ 
exercises. Thus, the logic of incorporating split-style Olympic weightlifting exercises into a volleyball strength training program can be further strengthened.

In our study, the highest adaptation was observed at $5-\mathrm{m}$ sprint time performance $(d=1.54)$. A significant improvement was observed in the $20-\mathrm{m}$ sprint time performance $(d=0.81)$. The difference in the magnitude of this effect between the sprint start phase and the sprint acceleration phase can be considered as an important finding. Similar studies may be further conducted because the sprint time is generally evaluated over a 40 -yard distance. Studies have shown strong relationships between weightlifting movements and sprint [5] and COD [19]. Hoffman et al. [34] compared their weight lifting and power lifting trainings programs and found a $175 \%$ improvement in their 40-yard distance. Tricoli et al. [26] reported an improvement in sprinting performance after an 8-week training intervention, which was performed thrice a week for a weightlifting group compared with the vertical jumping training group. Our research strengthens the findings of these studies.

In our study, SW exercises were found to increase LS. Although Olympic weightlifting and its derivatives may be the basis of a stiffness program as expert opinion [35], no study investigated the effect of Olympic weightlifting training and their derivatives on LS. Because stiffness is reported to increase with running velocity and vertical jump, most researchers believe that stiffness should be increased to improve sports performance [36]. In a study measuring leg and joint stiffness, trained athletes have been shown to have more leg stiffness than their counterparts in the general population [37]. In a similar study, the same authors found that power athletes had more leg stiffness than endurance training athletes [38]. This suggests that, in particular, where the efficient power transmission is important for the task, the rigidity of the force transmission is important. Similar research is needed to reinforce the findings of stiffness.

This study has some limitations, which have to be pointed out. First, an important limitation of our study is the difficulty in finding clearer results owing to the lack of an $\mathrm{CW}$ group. Thus, more explicit relationships could be detected on the measured variables of $\mathrm{SW}$ and $\mathrm{CW}$ exercises, particularly on SJ. Second, the study only had a 6-week training period. Although we performed adaptation training for 2 weeks of SW exercises, the fact that the athletes would take a one-week break because of the national holiday prevented us from continuing the actual training period for more weeks. Future research will be able to perform much stronger research by removing these limitations.

Practically, our study employed a 2-week adaptation program and SW training for 6 weeks; however, the data on sprinting, stiffness, and COD are important findings to consider for trainers and athletes to add SW exercises to volleyball strength training programs particularly because of their impact on SJ performance. In addition, SW exercises seem to allow for a comprehensive physical development in a short time. Therefore, these exercises are recommended for use in volleyball and other sports strength training. Although the sample of our study consisted of only female collegiate volleyball players, the results can be generalized to other sports who require leg stiffness, sprint, COD, and vertical jump activities.

\section{Conclusions}

This study was the first to investigate the effectiveness of SW exercises in female collegiate volleyball players. We have demonstrated that SW training (twice a week for $6 \mathrm{wk}$ ) improve $5 \mathrm{Ms}$, 20Ms, LS, COD, and SJ in collegiate female volleyball players. Further studies of $\mathrm{CW}$ groups are required for better evaluation of the present research finding.

\section{Acknowledgements}

The authors would like to thank all of the athletes for their participation in the study.

\section{REFERENCES}

[1] M. Allen Hedrick, "Training for high level performance in women's collegiate volleyball: Part I training requirements," Strength and Conditioning Journal, vol. 29, no. 6, p. 50, 2007.

[2] J. M. Sheppard, T. Gabbett, K.-L. Taylor, J. Dorman, A. J. Lebedew, and R. Borgeaud, "Development of a repeated-effort test for elite men's volleyball," International Journal of Sports Physiology and Performance, vol. 2, no. 3, pp. 292-304, 2007.

[3] P. M. Holmberg, "Weightlifting to Improve Volleyball Performance," Strength \& Conditioning Journal, vol. 35, no. 2, pp. 79-88, 2013.

[4] J. L. Ayers, M. DeBeliso, T. G. Sevene, and K. J. Adams, "Hang cleans and hang snatches produce similar improvements in female collegiate athletes," Biology of Sport, vol. 33, no. 3, pp. 251-256, 05/10

[5] N. Hori, R. U. Newton, W. A. Andrews, N. Kawamori, M. R. McGuigan, and K. Nosaka, "Does performance of hang power clean differentiate performance of jumping, sprinting, and changing of direction?," The Journal of Strength \& Conditioning Research, vol. 22, no. 2, pp. 412-418, 2008.

[6] G. G. Haff, A. Whitley, and J. A. Potteiger, "A brief review: Explosive exercises and sports performance," Strength \& Conditioning Journal, vol. 23, no. 3, p. 13, 2001.

[7] T. J. Suchomel, P. Comfort, and J. P. Lake, "Enhancing the Force-Velocity Profile of Athletes Using Weightlifting Derivatives," Strength \& Conditioning Journal, vol. 39, no. 
1, pp. 10-20, 2017.

[8] P. Cormie, M. R. McGuigan, and R. U. Newton, "Developing maximal neuromuscular power," Sports medicine, vol. 41, no. 1, pp. 17-38, 2011.

[9] L. Z. Chiu and B. K. Schilling, "A primer on weightlifting: From sport to sports training," Strength and Conditioning journal, vol. 27, no. 1, p. 42, 2005.

[10] P. Comfort, M. Allen, and P. Graham-Smith, "Kinetic comparisons during variations of the power clean," The Journal of Strength \& Conditioning Research, vol. 25, no. 12, pp. 3269-3273, 2011.

[11] J. Duba, W. J. Kraemer, and G. Martin, "Progressing from the hang power clean to the power clean: A 4-step model," Strength \& Conditioning Journal, vol. 31, no. 3, pp. 58-66, 2009.

[12] J. Garhammer, "A comparison of maximal power outputs between elite male and female weightlifters in competition," International Journal of Sport Biomechanics, vol. 7, no. 1, pp. 3-11, 1991.

[13] L. Ruggiero, S. Dewhurst, and T. M. Bampouras, "Validity and reliability of two field-based leg stiffness devices: implications for practical use," Journal of applied biomechanics, vol. 32, no. 4, pp. 415-419, 2016.

[14] G. Dalleau, A. Belli, F. Viale, J.-R. Lacour, and M. Bourdin, "A simple method for field measurements of leg stiffness in hopping," International journal of sports medicine, vol. 25, no. 03, pp. 170-176, 2004.

[15] T. Sattler, D. Sekulic, V. Hadzic, O. Uljevic, and E. Dervisevic, "Vertical jumping tests in volleyball: reliability, validity, and playing-position specifics," The Journal of Strength \& Conditioning Research, vol. 26, no. 6, pp. 1532-1538, 2012.

[16] M. Brzycki, "Strength testing-predicting a one-rep max from reps-to-fatigue," Journal of Physical Education, Recreation \& Dance, vol. 64, no. 1, pp. 88-90, 1993.

[17] W. Hopkins, S. Marshall, A. Batterham, and J. Hanin, "Progressive statistics for studies in sports medicine and exercise science," Medicine+ Science in Sports + Exercise, vol. 41, no. 1, p. 3, 2009.

[18] M. R. Rhea, "Determining the magnitude of treatment effects in strength training research through the use of the effect size," Journal of strength and conditioning research, vol. 18, pp. 918-920, 2004.

[19] J. M. Carlock et al., "The relationship between vertical jump power estimates and weightlifting ability: a field-test approach," The Journal of Strength \& Conditioning Research, vol. 18, no. 3, pp. 534-539, 2004.

[20] D. Hackett, T. Davies, N. Soomro, and M. Halaki, "Olympic weightlifting training improves vertical jump height in sportspeople: a systematic review with meta-analysis," $\mathrm{Br} J$ Sports Med, pp. bjsports-2015-094951, 2015.

[21] T. R. Andrews, T. Mackey, T. A. Inkrott, S. R. Murray, I. E. Clark, and R. W. Pettitt, "Effect of hang cleans or squats paired with countermovement vertical jumps on vertical displacement," The Journal of Strength \& Conditioning Research, vol. 25, no. 9, pp. 2448-2452, 2011.
[22] M. R. McCann and S. P. Flanagan, "The effects of exercise selection and rest interval on post-activation potentiation of vertical jump performance," The Journal of Strength \& Conditioning Research, vol. 24, no. 5, pp. 1285-1291, 2010.

[23] F. Arabatzi, E. Kellis, and E. S.-S. De Villarreal, "Vertical jump biomechanics after plyometric, weight lifting, and combined (weight lifting+ plyometric) training," The Journal of Strength \& Conditioning Research, vol. 24, no. 9, pp. 2440-2448, 2010.

[24] B. T. Channell and J. Barfield, "Effect of Olympic and traditional resistance training on vertical jump improvement in high school boys," The Journal of Strength \& Conditioning Research, vol. 22, no. 5, pp. 1522-1527, 2008.

[25] W. H. Otto III, J. W. Coburn, L. E. Brown, and B. A. Spiering, "Effects of weightlifting vs. kettlebell training on vertical jump, strength, and body composition," The Journal of Strength \& Conditioning Research, vol. 26, no. 5, pp. 1199-1202, 2012.

[26] V. Tricoli, L. Lamas, R. Carnevale, and C. Ugrinowitsch, "Short-term effects on lower-body functional power development: weightlifting vs. vertical jump training programs," The Journal of Strength \& Conditioning Research, vol. 19, no. 2, pp. 433-437, 2005.

[27] M. Stone, R. Byrd, J. Tew, and M. Wood, "Relationship between anaerobic power and olympic weightlifting performance," The Journal of sports medicine and physical fitness, vol. 20, no. 1, p. 99, 1980.

[28] C. Helland et al., "Training Strategies to Improve Muscle Power: Is Olympic-style Weightlifting Relevant?," Med Sci Sports Exerc, vol. 49, no. 4, pp. 736-745, Apr 2017.

[29] [29] T. J. Suchomel, P. Comfort, and M. H. Stone, "Weightlifting pulling derivatives: rationale for implementation and application," Sports Med, vol. 45, no. 6, pp. 823-39, Jun 2015.

[30] G. G. Haff and S. Nimphius, "Training principles for power," Strength \& Conditioning Journal, vol. 34, no. 6, pp. 2-12, 2012.

[31] M. H. Stone, W. A. Sands, and M. E. Stone, "Weightlifting: program design," Strength and Conditioning Journal, vol. 28 , no. 2 , p. $10,2006$.

[32] A. C. o. S. Medicine, "American College of Sports Medicine position stand. Progression models in resistance training for healthy adults," Medicine and science in sports and exercise, vol. 41, no. 3, p. 687, 2009.

[33] A. Storey and H. K. Smith, "Unique aspects of competitive weightlifting: performance, training and physiology," Sports Med, vol. 42, no. 9, pp. 769-90, Sep 012012.

[34] J. R. Hoffman, J. Cooper, M. Wendell, and J. Kang, "Comparison of Olympic vs. traditional power lifting training programs in football players," The Journal of Strength \& Conditioning Research, vol. 18, no. 1, pp. 129-135, 2004.

[35] J. Brazier, C. Bishop, C. Simons, M. Antrobus, P. J. Read, and A. N. Turner, "Lower extremity stiffness: Effects on performance and injury and implications for training," Strength \& Conditioning Journal, vol. 36, no. 5, pp. 103-112, 2014. 
[36] M. Brughelli and J. Cronin, "Influence of running velocity on vertical, leg and joint stiffness," Sports Medicine, vol. 38, no. 8, pp. 647-657, 2008.

[37] H. Hobara et al., "Differences in lower extremity stiffness between endurance-trained athletes and untrained subjects," Journal of Science and Medicine in Sport, vol. 13, no. 1, pp. 106-111, 2010.

[38] H. Hobara et al., "Determinants of difference in leg stiffness between endurance-and power-trained athletes," Journal of biomechanics, vol. 41, no. 3, pp. 506-514, 2008. 